\title{
Diagnostic Validity of an Automated Probabilistic Tractography in Amnestic Mild Cognitive Impairment
}

\author{
Won Sang Jung ${ }^{1}$, Yoo Hyun Um², Dong Woo Kang ${ }^{3}$, Chang Uk Lee ${ }^{3}$, Young Sup Woo ${ }^{4}$, Won-Myong Bahk ${ }^{4}$, \\ Hyun Kook Lim $^{4}$ \\ Departments of ${ }^{1}$ Radiology and ${ }^{2}$ Psychiatry, St. Vincent Hospital, Suwon, ${ }^{3}$ Department of Psychiatry, Seoul St. Mary's Hospital, College of \\ Medicine, The Catholic University of Korea, Seoul, ${ }^{4}$ Department of Psychiatry, Yeouido St. Mary's Hospital, College of Medicine, The Catholic \\ University of Korea, Seoul, Korea
}

\begin{abstract}
Objective: Although several prior works showed the white matter (WM) integrity changes in amnestic mild cognitive impairment (aMCl) and Alzheimer's disease, it is still unclear the diagnostic accuracy of the WM integrity measurements using diffusion tensor imaging (DTI) in discriminating aMCl from normal controls. The aim of this study is to explore diagnostic validity of whole brain automated probabilistic tractography in discriminating aMCl from normal controls. Methods: One hundred-two subjects (50 aMCl and 52 normal controls) were included and underwent DTI scans. Whole brain WM tracts were reconstructed with automated probabilistic tractography. Fractional anisotropy (FA) and mean diffusivity (MD) values of the memory related WM tracts were measured and compared between the $\mathrm{aMCl}$ and the normal control groups. In addition, the diagnostic validities of these WM tracts were evaluated.

Results: Decreased FA and increased MD values of memory related WM tracts were observed in the aMCI group compared with the control group. Among FA and MD value of each tract, the FA value of left cingulum angular bundle showed the highest area under the curve (AUC) of 0.85 with a sensitivity of $88.2 \%$, a specificity of $76.9 \%$ in differentiating $\mathrm{MCl}$ patients from control subjects. Furthermore, the combination FA values of WM integrity measures of memory related WM tracts showed AUC value of 0.98 , a sensitivity of $96 \%$, a specificity of $94.2 \%$.

Conclusion: Our results with good diagnostic validity of WM integrity measurements suggest DTI might be promising neuroimaging tool for early detection of $\mathrm{aMCl}$ and $\mathrm{AD}$ patients.
\end{abstract}

KEY WORDS: Mild cognitive impairment; Diffusion tensor imaging; Diffusion tensor imaging; Biomarker.

\section{INTRODUCTION}

The incidence of dementia and cognitive disorder is increasing. ') Amnestic mild cognitive impairment (aMCl) is a selective decline in memory in the situation of otherwise normal cognition and normal daily functioning. ${ }^{2)}$ The rate of change from $\mathrm{MCl}$ to overt dementia is substantial at $10 \%$ to $15 \%$ per year, the majority of these being Alzheimer's disease (AD). ${ }^{2-4)}$ Therapeutic approach for $A D$ is more focused on preventing or delaying of disease onset in preclinical stage. ${ }^{5)}$ Therefore, many inves-

Received: November 7, 2016/Revised: November 15, 2016 Accepted: November 22, 2016

Address for correspondence: Hyun Kook Lim, MD, PhD Department of Psychiatry, Yeouido St. Mary's Hospital, College of Medicine, The Catholic University of Korea, Seoul, Korea Tel: +82-2-3779-1048, Fax: +82-2-780-6577

E-mail:drblues@catholic.ac.kr

ORCID: https://orcid.org/0000-0001-8742-3409 tigators are striving to establish efficient biomarkers for early detection of AD for preventive interventions. ${ }^{6-8)}$

The diffusion tensor imaging (DTI) is an in vivo magnetic resonance imaging (MRI) technique, which is able to evaluate the microstructural integrity of white matter (WM). ${ }^{9)}$ DTI is a non-invasive imaging method based on the diffusion characteristics of water molecules. ${ }^{10)}$ The results of previous studies about $\mathrm{aMCl}$ and $\mathrm{AD}$ patients with DTI analysis showed microstructural changes of WM in brain. ${ }^{11-15)}$ The majority of findings in previous DTI studies was increased mean diffusivity (MD) and reduced fractional anisotropy (FA) in parietal and temporal lobes, which is located posterior hemispheric WM. Several studies with tractography based analysis more specifically showed WM structural abnormality in the splenium of the corpus callosum, posterior cingulum, uncinate fasciculi. $^{16-19)}$ The promising value of DTI as an imaging bio-

(ㄷ) This is an Open-Access article distributed under the terms of the Creative Commons Attribution Non-Commercial License (http://creativecommons.org/licenses/by-nc/4.0) which permits unrestricted non-commercial use, distribution, and reproduction in any medium, provided the original work is properly cited. 
marker for $\mathrm{AD}$ and $\mathrm{aMCl}$ has been reported. There were $\mathrm{a}$ few studies combining DTI and morphometry measures to increase diagnostic performance of aMCl patients. ${ }^{2,20,21)}$ Also, DTI was found to be more sensitive than hippocampal volumetry for distinguishing between aMCl patients and healthy controls. ${ }^{22)}$ In other studies, hippocampal diffusivity was a better predictor than hippocampal volumetry for the conversion from $\mathrm{MCl}$ to $A D^{23)}$ and WM integrity change of fornix was the earliest finding of cognitive impairment group in normal controls. ${ }^{24)}$ The results of these studies suggest that alterations of WM integrity can be a useful imaging biomarker for early prediction of cognitive impairment patients.

In previous studies of DTI in AD and aMCI patients, commonly used methodologies were quantitative regionof-interest (ROI) based techniques or a voxel-based analysis (VBA) or quantitative tract-based analysis techniques. The ROI placement by manual drawing method is not precisely reproducible in different cases and time consuming. ${ }^{25,26)}$ The VBA technique using statistical parametric mapping (SPM) has well-known problem of arbitrary spatial smoothing extent, which unavoidably affect the group differences. ${ }^{27}$ Recently, many DTI studies have applied tract-based spatial statistics (TBSS) in FSL (FMRIB Software Library, http://www.fmrib.ox.ac.uk/fsl/) software, which can overcome SPM problem, using an algorithm aligns each individuals mean FA to a common standard space in registration process. However, TBSS is more suitable tool for group analysis with carrying out voxelwise statistics across subjects, not convenient to reveal altered WM integrity of individual subject.

The purpose of this study was to investigate the diagnostic accuracy of automated tractography based on DTI for differentiation of aMCl patient from normal control group. We hypothesized diffusion metrics changes more obviously occur at the WM tracts related memory circuit such as inferior longitudinal fasciculus (ILF), cingulum, parietal bundle of superior longitudinal fasciculus, forcep major and minor of corpus callosum. ${ }^{28)}$

\section{METHODS}

\section{Subjects}

One hundred-two subjects took part in this study (50 with $\mathrm{aMCl}$ and 52 healthy elderly controls). They were recruited from the Catholic Geriatric Brain MRI Database which was built through the outpatient geriatric psychiatry clinic of St. Vincent's Hospital located in Suwon, South Korea from October 2009 to February 2016.

Diagnosis of aMCl was made according to the criteria by Petersen ${ }^{29)}$ including a subjective cognitive complaint (corroborated by an informant), impairment of memory with comprehensive neuropsychological test $(<1.5$ standard deviation below the performance of age and education control subjects in the Korean version of the Consortium to Establish a Registry for Alzheimer's Disease (CERAD-K) neuropsychological battery ${ }^{30}$ ), normal general cognitive functioning, and preserved instrumental activities of daily living. All aMCI individuals had an overall Clinical Dementia Rating (CDR) score of 0.5. ${ }^{31)}$ Fifty two healthy controls with an overall CDR of 0 were also selected after a standardized clinical assessment and CERAD-K neuropsychological test battery. A clinical neuroradiologist (WSJ) examined the brain MRIs of all the subjects; no gross abnormalities were reported in any participant and showed normal-appearing WM.

All participants gave written informed consent, and the study was approved by the Ethical Committee of the local Institutional Review Board of the Catholic University of Korea (No. VC15EISI0044). All subjects were right-handed.

\section{Magnetic Resonance Imaging Acquisition}

All participants underwent MRI scans on a 3-tesla whole body scanner equipped with an 8-channel phasedarray head coil (Verio; Siemens, Erlangen, Germany). The scanning parameters of the T1-weighted three-dimensional magnetization-prepared rapid gradient echo sequences were as follows; echo time $=2.5 \mathrm{~ms}$, repetition time $=1,900 \mathrm{~ms}$, inversion time $=900 \mathrm{~ms}$, flip angle $=9^{\circ}$, field of view $=250 \times 250 \mathrm{~mm}$, matrix $=256 \times 256$, and voxel size $=1.0 \times 1.0 \times 1.0 \mathrm{~mm}$. In addition the scanning parameters of the DTI sequences were as follows; echo planar imaging, $T R=9,300 \mathrm{~ms}, \mathrm{TE}=94 \mathrm{~ms}$, field of view=192 mm, voxel dimension $=2 \mathrm{~mm}$ isotropic, $\mathrm{B}$-value $=1,000$, gradients applied $=30$ isotropically, and distributed and acquisition time $=21 \mathrm{~min}$.

\section{Image Processing}

The workflow of imaging processing can be summarized in five steps.

1) Cortical and subcortical segmentations of structural T1-weighted MRI by automated reconstruction tool of 
FreeSurfer software (version 5.3.0; https://surfer.nmr. mgh.harvard.edu). The technical details had been well described in previous articles. ${ }^{32-34)}$ Briefly, this process consists of a Talairach transform of each subject's native brain, removal of the skull, and segmentation of the gray and WM tissue. This is fundamental step for addressing specific anatomic parts of each subjects' brain.

2) Preprocessing of the DTI images. This process includes Eddy-current correction, intra-subject registration (diffusion weighted image to T1 data) by FreeSurfer's bbregister, ${ }^{35)}$ inter-subject registration of each subject to a common MNI152 template, ${ }^{36)}$ creation of cortical and WM masks from T1 reconstruction data, fitting of diffusion tensors using FSL's dtifit (http://www.fmrib.ox. ac.uk/fsl), computing anatomical priors for WM pathways from training data.

3) Ball and stick modeling ${ }^{37)}$ of the diffusion data using FSL's bedpostx tool. This model provides one isotropic diffusion and multiple anisotropic diffusion compartments per voxel, revealing the diffusion data of voxel as a volume and orientations.

4) Automated reconstructing WM pathways. TRACULA (TRActs Constrained by UnderLying Anatomy, https://surfer.nmr.mgh.harvard.edu/fswiki/Tracula) was introduced as an automated analyzing method for WM tract evaluation. ${ }^{38)}$ TRACULA utilizes formerly obtained anatomic knowledge from a set of training participants

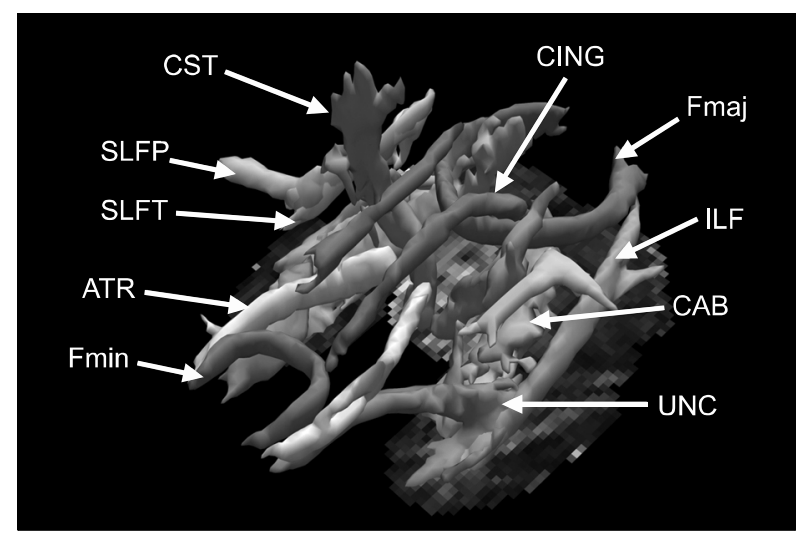

Fig. 1. White matter tracts reconstructed by automated probabilistic tractography.

ATR, anterior thalamic radiations; $\mathrm{CAB}$, cingulum-angular bundle; CING, cingulum-cingulate gyrus bundle; CST, corticospinal tract; Fmaj, corpus callosum-forceps major; Fmin, corpus callosum-forceps minor; ILF, inferior longitudinal fasciculus; SLFP, superior longitudinal fasciculus-parietal terminations; SLFT, superior longitudinal fasciculustemporal terminations; UNC, uncinate fasciculus. where the WM tracts were labeled manually. This prior information is the probability of each tract to travel through or next to each of the cortical and subcortical segmentation labels from FreeSurfer. Based on this data, TRACULA can reconstruct 18 major WM tracts from local diffusion orientation of each subject's ball and stick model. The 18 available WMs are corticospinal tract (CST), ILF, uncinate fasciculus, anterior thalamic radiation (ATR), cingulum-cingulate gyrus (supracallosal) bundle (CING), cingulum-angular (infracallosal) bundle (CAB), superior longitudinal fasciculus-parietal bundle (SLFP), superior longitudinal fasciculus-temporal bundle (SLFT), corpus callosum-forceps major (Fmaj), corpus callosum-forceps minor (Fmin). The output of TRACULA is a probabilistic distribution for each of the 18 tracts (Fig. 1). ${ }^{38)}$

5) Obtaining value of diffusion metrics for statistical analysis. We extracted statistics result files of each WM tracts, containing various diffusion measures such as tract volume, FA, MD. As the motor cortex and related WM tracts are known to be not affected by $A D$ at the earlier stage, the results of both CSTs, well-known WM tract related motor function, were omitted for statistical analysis.

\section{Statistical Analysis}

Statistical analyses for demographic data (Table 1) were performed with the Statistical Package for Social Sciences

Table 1. Demographic and clinical characteristics of study participants

\begin{tabular}{lccc}
\hline \multicolumn{1}{c}{ Characteristic } & $\begin{array}{c}\text { Control group } \\
(\mathrm{n}=52)\end{array}$ & $\begin{array}{c}\text { aMCl group } \\
(\mathrm{n}=50)\end{array}$ & $p$ value \\
\hline Age (yr) & $69.2 \pm 6.4$ & $71.1 \pm 6.9$ & $\mathrm{NS}$ \\
Education (yr) & $10.4 \pm 4.4$ & $9.7 \pm 3.2$ & $\mathrm{NS}$ \\
Sex (male:female) & $20: 32$ & $22: 28$ & $\mathrm{NS}$ \\
CERAD-K battery & & & \\
Verbal fluency & $13.9 \pm 3.9$ & $12.2 \pm 3.5$ & $\mathrm{NS}$ \\
BNT & $12.7 \pm 2.4$ & $11.4 \pm 2.3$ & $\mathrm{NS}$ \\
MMSE & $28.4 \pm 1.5$ & $25.4 \pm 2.3$ & $<0.0001$ \\
Word list memory & $18.5 \pm 4.5$ & $7.0 \pm 3.4$ & $<0.0001$ \\
Constructional praxis & $9.4 \pm 1.5$ & $8.7 \pm 1.9$ & $\mathrm{NS}$ \\
Word list recall & $7.7 \pm 1.8$ & $2.3 \pm 1.8$ & $<0.0001$ \\
Word list recognition & $9.9 \pm 1.2$ & $6.5 \pm 1.7$ & $<0.0001$ \\
Constructional recall & $6.9 \pm 2.9$ & $3.2 \pm 2.9$ & $<0.0001$ \\
\hline
\end{tabular}

Values are presented as mean \pm standard deviation or number only. aMCl, amnestic mild cognitive impairment; NS, not significant; CERAD-K, the Korean version of Consortium to Establish a Registry for Alzheimer's Disease; BNT, 15-item Boston Naming Test; MMSE, Mini Mental Status Examination. 
software (version 12.0; SPSS Inc., Chicago, IL, USA). Assumptions for normality were tested for all continuous variables. Normality was tested using the KolmogorovSmirnov test. All variables were normally distributed. The independent $t$-test and the $\chi^{2}$ test were used to assess potential differences between the a $\mathrm{MCl}$ groups and healthy control groups for all demographic variables. All statistical analyses had a two-tailed a level of $<0.05$ for defining statistical significance.

The general linear model was implemented to identify the of $16 \mathrm{WM}$ tracts in which aMCI subjects showed significant differences in mean values of DTI parameters (FA, $\mathrm{MD}$ ) relative to controls. The effects of age, education, total intracranial volume, and gender were regressed out in these models. The Bonferroni method was used for correction for multiple comparisons. Statistical significance was thresholded at Bonferroni corrected $p<0.05$.

We performed binary logistic regression with receiver operator characteristic (ROC) analysis to evaluate the sensitivity, specificity, and accuracy of DTI parameters (FA, $\mathrm{MD}$ ) of each $16 \mathrm{WM}$ tracts to discriminate $\mathrm{aMCl}$ from the controls, and predict the aMCI subjects who converted to $A D$. Area under the curve (AUC) was used to evaluate the optimal cutoff point. A result with $p<0.05$ and AUC value $>0.5$ were considered statistically significant and diagnostically meaningful. We also calculated the specificity and sensitivity of each DTI parameters of 'combined'

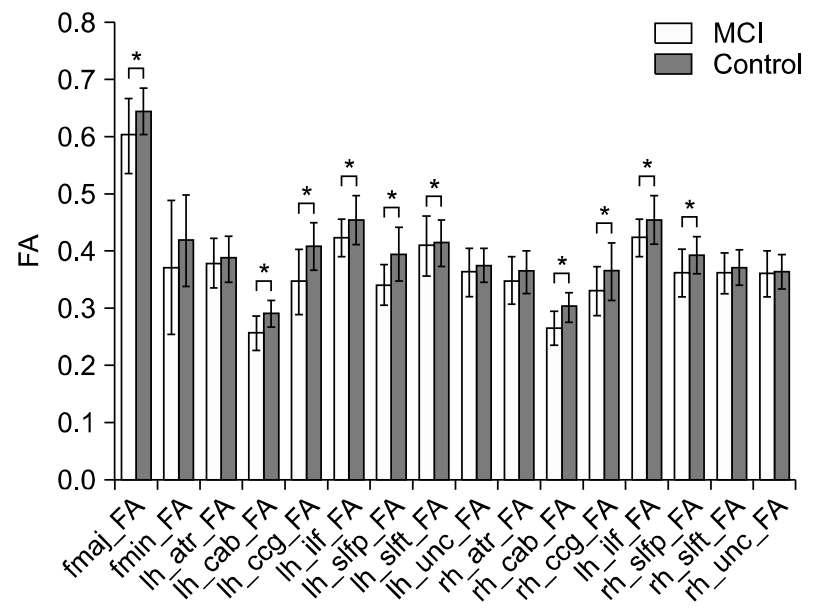

measurements of previously known memory related circuits (Fmaj, CAB, CING, SLFP, and ILF) ${ }^{28,39)}$ were indicated by areas under the ROC curve.

\section{RESULTS}

\section{Demographic and Clinical Characteristics}

Table 1 shows the baseline demographic data for the subject groups. There was no significant difference in sex, age and education between the $\mathrm{aMCl}$ group and the control group. Compared with the controls, patients with $\mathrm{aMCl}$ showed significantly poorer performances on the Mini Mental State Examination, word list memory, word list recall, word list recognition, and constructional recall in the CERAD-K neuropsychological test $(p<0.05)$.

\section{Between-group Differences in WM Integrity of the $\mathbf{1 6}$ a Priori WM Tracts}

Group comparison results of FA and MD values between the $\mathrm{aMCl}$ and the normal control group are shown in Figure 2.

Among 16 different WM tracts, 10 tracts (Fmaj, both CABs, both CINGs, both ILFs, both SLFPs and left SLFT) of $\mathrm{aMCl}$ group showed statistically significantly lower FA values than normal control group after age, sex, and total intracranial volumes corrected. In addition, $13 \mathrm{WM}$ tracts of 16 tracts showed statistically significantly increased

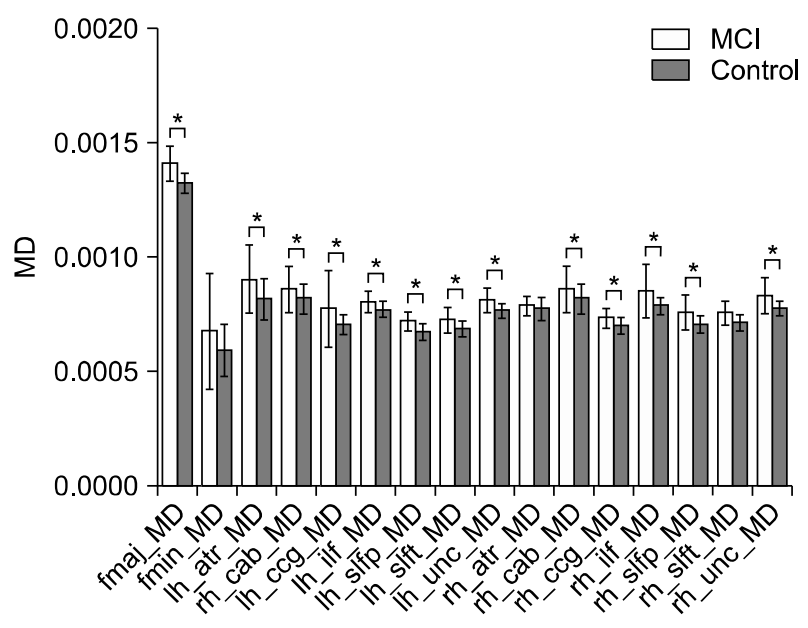

Fig. 2. Group comparison results of fractional anisotropy (FA) and mean diffusivity (MD) values between the amnestic mild cognitive impairment (aMCl) and the normal control groups.

fmaj, corpus callosum-forceps major; fmin, corpus callosum-forceps minor; atr, anterior thalamic radiations; cab, cingulum-angular bundle; ccg, cingulum gyrus bundle; slfp, superior longitudinal fasciculus-parietal terminations; ilf, inferior longitudinal fasciculus; slft, superior longitudinal fasciculus-temporal terminations; unc, uncinate fasciculus; rh, right hemisphere; lh, left hemisphere.

*Statistically significant after age, sex, and total intracranial volumes corrected. 
Table 2. Sensitivity and specificity to predict aMCl with measurement of 5 memory related white matter (WM) tracts

\begin{tabular}{|c|c|c|c|c|c|}
\hline $\begin{array}{l}\text { WM } \\
\text { tracts }\end{array}$ & $\begin{array}{c}\text { Sensitivity } \\
(\%)\end{array}$ & $\begin{array}{l}\text { Specificity } \\
(\%)\end{array}$ & $\begin{array}{l}\text { PPV } \\
(\%)\end{array}$ & $\begin{array}{l}\text { NPV } \\
(\%)\end{array}$ & AUC \\
\hline \multicolumn{6}{|l|}{ Fmaj } \\
\hline FA & 72.1 & 61.5 & 64.3 & 61.7 & 0.70 \\
\hline MD & 82.1 & 78.8 & 78.8 & 82.0 & 0.84 \\
\hline \multicolumn{6}{|l|}{ CAB-left } \\
\hline FA & 88.2 & 76.9 & 78.6 & 87.0 & 0.85 \\
\hline MD & 80.7 & 71.2 & 72.7 & 78.7 & 0.76 \\
\hline \multicolumn{6}{|c|}{ CAB-right } \\
\hline FA & 88.0 & 76.8 & 78.6 & 87.0 & 0.83 \\
\hline MD & 76.0 & 53.8 & 61.3 & 70.0 & 0.65 \\
\hline \multicolumn{6}{|c|}{ CING-left } \\
\hline FA & 84.0 & 69.2 & 72.4 & 81.8 & 0.81 \\
\hline $\mathrm{MD}$ & 66.0 & 78.8 & 75.0 & 70.7 & 0.72 \\
\hline \multicolumn{6}{|c|}{ CING-right } \\
\hline FA & 74.0 & 61.5 & 64.9 & 71.1 & 0.71 \\
\hline MD & 70.0 & 67.3 & 66.7 & 68.6 & 0.71 \\
\hline \multicolumn{6}{|c|}{ SLFP-left } \\
\hline FA & 80.0 & 71.1 & 72.7 & 78.7 & 0.82 \\
\hline $\mathrm{MD}$ & 78.0 & 67.3 & 69.6 & 76.1 & 0.79 \\
\hline \multicolumn{6}{|c|}{ SLFP-right } \\
\hline FA & 72.0 & 69.2 & 69.2 & 72.0 & 0.72 \\
\hline $\mathrm{MD}$ & 70.0 & 67.3 & 67.3 & 70.0 & 0.74 \\
\hline \multicolumn{6}{|l|}{ ILF-left } \\
\hline FA & 72.0 & 76.9 & 75.0 & 74.1 & 0.74 \\
\hline MD & 74.0 & 61.5 & 64.9 & 71.1 & 0.70 \\
\hline \multicolumn{6}{|l|}{ ILF-right } \\
\hline FA & 72.0 & 84.6 & 81.8 & 75.9 & 0.80 \\
\hline $\mathrm{MD}$ & 70.0 & 67.3 & 67.3 & 70.0 & 0.74 \\
\hline \multicolumn{6}{|c|}{ Sum of 9 tracts } \\
\hline FA & 96.0 & 94.2 & 94.1 & 96.1 & 0.98 \\
\hline MD & 84.0 & 92.3 & 91.3 & 85.7 & 0.95 \\
\hline
\end{tabular}

aMCl, amnestic mild cognitive impairment; PPV, positive predictive value; NPV, negative predictive value; AUC, area under the receiver operating characteristic curve; $F A$, fractional anisotropy; $\mathrm{MD}$, mean diffusivity; Fmaj, forceps major; $\mathrm{CAB}$, cingulum-angular bundle; CING, cingulum-cingulate gyrus; SLFP, superior longitudinal fasciculus; ILF, inferior longitudinal fasciculus.

$\mathrm{MD}$ values in aMCl group, except 3 tracts (Fmin, right ATR and right SLFT). There were no statistical differences of $\mathrm{FA}$ and $\mathrm{MD}$ values of the CST between the $\mathrm{aMCl}$ group and the control group.

\section{Diagnostic Performance and Receiver Operating Characteristic Curve Analysis}

The diagnostic performances of selected $9 \mathrm{WM}$ tracts (Fmaj, both CABs, both CINGs, both SLFPs and both ILFs) and sum of these tracts are shown in Table 2. Among FA and $M D$ value of each tract, the FA value of left $C A B$ showed highest AUC of 0.85 with a sensitivity of $88.2 \%$, a specificity of $76.9 \%$, a positive predictive value (PPV) of

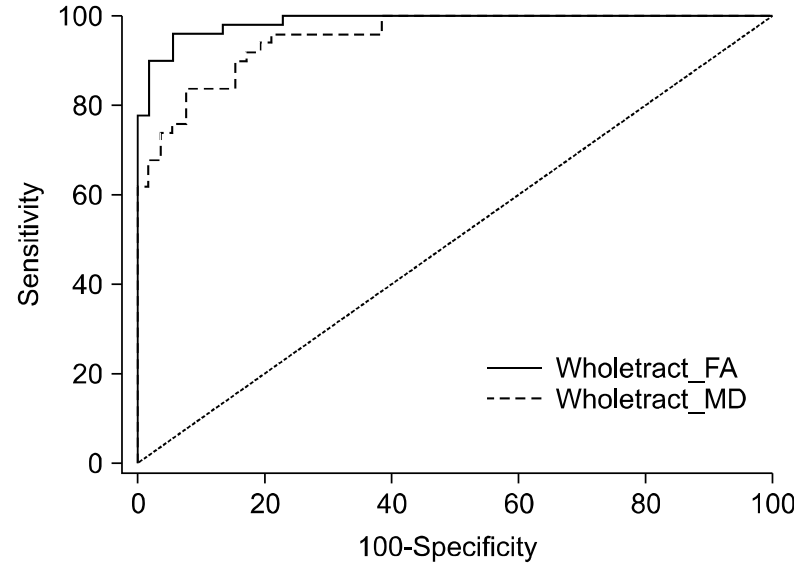

Fig. 3. Receiver operator characteristic curve analysis of fractional anisotropy (FA) and mean diffusivity (MD) values of memory related tracts (Fmaj, CAB, CING, SLFP, and ILF) between the amnestic mild cognitive impairment and the normal control groups.

Fmaj, corpus callosum-forceps major; $\mathrm{CAB}$, cingulum-angular bundle; CING, cingulum-cingulate gyrus bundle; SLFP, superior longitudinal fasciculus-parietal terminations; ILF, inferior longitudinal fasciculus.

$78.6 \%$ and a negative predictive value (NPV) of $87 \%$. The $M D$ value of right $C A B$ showed lowest $A \cup C$ value of 0.65 with a sensitivity of $76 \%$, a specificity of $53.8 \%$, a PPV of $61.3 \%$ and a NPV of $70 \%$. The ROC curve analysis of sum of 9 tracts is shown in Figure 3. The FA value of sum of 9 tracts showed AUC value of 0.98 , a sensitivity of $96 \%$, a specificity of $94.2 \%$, a PPV of $94.1 \%$ and a NPV of $96.1 \%$. The MD value of sum of 9 tracts showed AUC value of 0.95 , a sensitivity of $84 \%$, a specificity of $92.3 \%$, a PPV of $91.3 \%$ and a NPV of $85.7 \%$.

\section{DISCUSSION}

To the best of our knowledge, this is the first study on diagnostic validity of automated probabilistic tract specific analysis in discriminating aMCl from normal controls.

We analyzed DTI data of normal control and MCI patient group using automated technique with probabilistic tractography, TRACULA. There are two different conceptions of tractography methods; deterministic and probabilistic tractography. ${ }^{25}$ Deterministic method is based on the assumption of the eigenvector is parallel to the underlying dominant fiber direction in each image voxel. Then, this algorithm propagates a single pathway form a seed point. ${ }^{40)}$ This method has a critical disadvantage of limited tracing a tract with crossing fibers, more than two different directional tracts in a voxel. The 
probabilistic method has been developed to attempt to resolve fiber crossings voxel and to model uncertainty. ${ }^{37)}$ TRACULA performs global probabilistic tractography of 18 major WM pathways of brain with using prior information on tract anatomy from a set of training subjects. It constrains WM pathways in new subjects based on this prior anatomical knowledge. We attempted to get more reproducible result by adopting automated technique.

There was a previous study about DTI of normal control, $\mathrm{MCl}$ and $\mathrm{AD}$ patients using TRACULA analysis. ${ }^{41)}$ They found out that several major WM tracts, especially cingulum-angular bundle, showed significant deterioration of WM integrity in $\mathrm{MCl}$ and AD patients. They demonstrated those WM alterations were observed even after correction of hippocampal volume. That could mean WM change in $\mathrm{MCl}$ and $\mathrm{AD}$ occurs not entirely dependent on hippocampal atrophic change. Gray matter pathology was not preceding condition of WM degeneration was observed in previous neuropathological study, ${ }^{42)}$ more assigned to vascular disease. These studies support our attempt to investigate diagnostic accuracy of single DTI modality for aMCI patients without analyzing gray matter atrophic change.

Our results demonstrated WM degradations of multiple major WM tracts in aMCI with difference of $\mathrm{MD}$ value (in 13 tracts) and FA value (in 10 tracts). Especially, we found out that each memory-related tract showed good diagnostic performance in differentiation $\mathrm{aMCl}$ patient from normal control group with 0.65 to 0.85 range of AUC. Among those tracts, cingulum showed overall the best diagnostic performance as a single tract, similar to previous TRACULAR study of $\mathrm{aMCl}$ and $\mathrm{AD} .^{41)}$ The cingulum is known as a tract contains efferent fiber from entorhinalhippocampal complex, ${ }^{43)}$ seems to reflect early alteration of aMCl patients. ${ }^{44)}$

In this study, we showed that combined FA and MD values of memory related tracts showed high diagnostic accuracy in both of FA and MD with 0.98, 0.95 of AUC respectively. Previously reported diagnostic performance of multimodal analysis with DTI and resting-state functional MRI (fMRI) was good, 96.3\% accuracy and area of 0.953 under the ROC curve in discriminating aMCI from normal controls. ${ }^{45)}$ In addition, another study also showed that combined use of FA value and cortical thickness of left temporal lobe improved the accuracy to diagnosing $\mathrm{MCl}$ compared with either measurement alone. ${ }^{21)}$ Our re- sults showed similar high diagnostic performance derived from single neuroimaging modality of DTI for differentiation of aMCl from healthy control group. To date, the medial temporal and hippocampus structural changes have been considered as pathological core of episodic memory impairment in the $\mathrm{AD}$ and $\mathrm{aMCl}^{43)}$ Moreover, prefrontal and retrosplenial cortex including posterior cingulate and precuneus structural and functional alterations were known to be involved in episodic memory impairment in $\mathrm{AD}$ and $\mathrm{aMCl}$. As several WM tracts such as Fmaj, Fmin, CAB, CING ILF, SLFP and SLFT are linking these memory related gray matter structures, WM integrity disruption of these tracts might be complexly related to the pathologic process of $\mathrm{aMCl}$ and $\mathrm{AD}$.

The tracts showed decreased WM integrity in our results were interestingly overlapped with previously reported regions of connectivity problem resting-state fMRI studies. Disruption of functional connectivity of default mode network was observed in previous studies ${ }^{46-49)}$ at posterior parietal cortex, the precuneus, posterior and anterior cingulated cortex, medial prefrontal cortex, hippocampus and thalamus. Further study using fMRI analysis and automated tractography is needed for better revealing of effective neuroimaging tool for aMCl patient.

Previous study showed result of FA was the least sensitive measurement among DTI indices such as FA, MD, radial diffusivity, axial diffusivity. ${ }^{13)}$ However, our results revealed remarkable change of FA value for differentiating $\mathrm{MCl}$ group from normal control group. It is unclear why there is such difference of diagnostic accuracy of FA value, however it could be derived from different methodology. They used ROI measurement method otherwise our DTI metrics were obtained from tractography of major WM tracts. Further comparison study about different methodology will necessary to explore these different results.

The limitations of our study were as follows. First, as we included the subjects with normal appearing WM in this study, we could not investigate the effect of apparent WM pathology on WM integrity change in aMCl subjects. Therefore, the results of this study should not be generalized to aMCI with other apparent WM pathology such as leukoaraiosis and microbleeding. Indeed, $\mathrm{aMCl}$ is a heterogeneous entity due to various pathological substrates and characterized by different outcomes. ${ }^{50)}$ Therefore, further study with pathological neuroimaging such as 
amyloid and tau positron emission tomography would increase more accurate diagnostic validity of DTI analysis in discriminating aMCI from normal controls. Second, as the TRACULA program does not provide the tractography of the fornix, we could not include the diagnostic validity of WM integrity of the fornix in this study. Several prior works showed the WM integrity of the fornix provided excellent diagnostic accuracy in discriminating $\mathrm{aMCl}$ and AD from normal controls. ${ }^{51)}$ In addition, unlike the other WM tracts, FA values from the fornix were not influenced by the noise of crossing fibers. ${ }^{52)}$ Hence, the inclusion of the fornix tract in the next version of TRACULA software would be increase validity of this program as AD biomarker analysis tool.

In conclusion, our results with aberrant WM integrity in the memory related WM tracts provided high diagnostic accuracy in discriminating $\mathrm{aMCl}$ from normal controls. These WM integrity changes suggest the automated probabilistic tractography methods might be promising neuroimaging tool for early detection of $\mathrm{aMCl}$ and $\mathrm{AD}$ patients. However, further larger studies will be needed to confirm real clinical benefits.

\section{Acknowledgments}

This research was supported by Basic Science Research Program through the National Research Foundation of Korea (NRF) funded by the Ministry of Science, ICT \& Future Planning (NRF-2015R1C1A1A02036578).

\section{REFERENCES}

1. Brodaty $\mathrm{H}$, Breteler MM, Dekosky ST, Dorenlot $\mathrm{P}$, Fratiglioni $\mathrm{L}$, Hock C, et al. The world of dementia beyond 2020. I Am Geriatr Soc 2011;59:923-927.

2. Cui Y, Wen W, Lipnicki DM, Beg MF, Jin JS, Luo S, et al. Automated detection of amnestic mild cognitive impairment in community-dwelling elderly adults: a combined spatial atrophy and white matter alteration approach. Neuroimage 2012;59:1209-1217.

3. Hu Z, Wu L, Jia J, Han Y. Advances in longitudinal studies of amnestic mild cognitive impairment and Alzheimer's disease based on multi-modal MRI techniques. Neurosci Bull 2014;30:198-206.

4. Petersen RC, Roberts RO, Knopman DS, Geda YE, Cha RH, Pankratz VS, et al. Prevalence of mild cognitive impairment is higher in men. The mayo clinic study of aging. Neurology 2010;75:889-897.

5. Citron M. Alzheimer's disease: strategies for disease modification. Nat Rev Drug Discov 2010;9:387-398.
6. Amlien IK, Fjell AM, Walhovd KB, Selnes P, Stenset V, Grambaite $\mathrm{R}$, et al. Mild cognitive impairment: cerebrospinal fluid tau biomarker pathologic levels and longitudinal changes in white matter integrity. Radiology 2013;266:295303.

7. Skillbäck T, Zetterberg H, Blennow K, Mattsson N. Cerebrospinal fluid biomarkers for Alzheimer disease and subcortical axonal damage in 5,542 clinical samples. Alzheimers Res Ther 2013;5:47.

8. Toledo JB, Zetterberg $\mathrm{H}$, van Harten AC, Glodzik L, Martinez-Lage $\mathrm{P}$, Bocchio-Chiavetto L, et al. Alzheimer's disease cerebrospinal fluid biomarker in cognitively normal subjects. Brain 2015;138:2701-2715.

9. Basser PJ, Mattiello J, LeBihan D. MR diffusion tensor spectroscopy and imaging. Biophys J 1994;66:259-267.

10. Bozzali M, Cherubini A. Diffusion tensor MRI to investigate dementias: a brief review. Magn Reson Imaging 2007;25: 969-977.

11. Bosch B, Arenaza-Urquijo EM, Rami L, Sala-Llonch R, Junqué C, Solé-Padullés $\mathrm{C}$, et al. Multiple DTI index analysis in normal aging, amnestic $\mathrm{MCl}$ and $A D$. Relationship with neuropsychological performance. Neurobiol Aging 2012;33:61-74.

12. Bozzali M, Padovani A, Caltagirone C, Borroni B. Regional grey matter loss and brain disconnection across Alzheimer disease evolution. Curr Med Chem 2011;18:2452-2458.

13. Nir TM, Jahanshad N, Villalon-Reina JE, Toga AW, Jack CR, Weiner MW, et al. Effectiveness of regional DTI measures in distinguishing Alzheimer's disease, $\mathrm{MCl}$, and normal aging. Neuroimage Clin 2013;3:180-195.

14. Pievani M, Agosta F, Pagani E, Canu E, Sala S, Absinta M, et al. Assessment of white matter tract damage in mild cognitive impairment and Alzheimer's disease. Hum Brain Mapp 2010; 31:1862-1875.

15. Stebbins GT, Murphy CM. Diffusion tensor imaging in Alzheimer's disease and mild cognitive impairment. Behav Neurol 2009;21:39-49.

16. Canu E, Agosta F, Spinelli EG, Magnani G, Marcone A, Scola $\mathrm{E}$, et al. White matter microstructural damage in Alzheimer's disease at different ages of onset. Neurobiol Aging 2013;34: 2331-2340.

17. Fischer FU, Scheurich A, Wegrzyn M, Schermuly I, Bokde AL, Klöppel S, et al. Automated tractography of the cingulate bundle in Alzheimer's disease: a multicenter DTI study. J Magn Reson Imaging 2012;36:84-91.

18. Morikawa M, Kiuchi K, Taoka T, Nagauchi K, Kichikawa K, Kishimoto T. Uncinate fasciculus-correlated cognition in Alzheimer's disease: a diffusion tensor imaging study by tractography. Psychogeriatrics 2010;10:15-20.

19. Yasmin H, Nakata Y, Aoki S, Abe O, Sato N, Nemoto K, et al. Diffusion abnormalities of the uncinate fasciculus in Alzheimer's disease: diffusion tensor tract-specific analysis using a new method to measure the core of the tract. Neuroradiology 2008;50:293-299. 
20. Müller MJ, Greverus D, Dellani PR, Weibrich C, Wille PR, Scheurich A, et al. Functional implications of hippocampal volume and diffusivity in mild cognitive impairment. Neuroimage 2005;28:1033-1042.

21. Wang L, Goldstein FC, Veledar E, Levey Al, Lah JJ, Meltzer CC, et al. Alterations in cortical thickness and white matter integrity in mild cognitive impairment measured by whole-brain cortical thickness mapping and diffusion tensor imaging. AJNR Am J Neuroradiol 2009;30:893-899.

22. Müller MJ, Greverus D, Weibrich C, Dellani PR, Scheurich A, Stoeter $\mathrm{P}$, et al. Diagnostic utility of hippocampal size and mean diffusivity in amnestic MCl. Neurobiol Aging 2007;28: 398-403.

23. Fellgiebel A, Dellani PR, Greverus D, Scheurich A, Stoeter $P$, Müller MJ. Predicting conversion to dementia in mild cognitive impairment by volumetric and diffusivity measurements of the hippocampus. Psychiatry Res 2006;146:283-287.

24. Fletcher E, Raman M, Huebner P, Liu A, Mungas D, Carmichael $\mathrm{O}$, et al. Loss of fornix white matter volume as a predictor of cognitive impairment in cognitively normal elderly individuals. JAMA Neurol 2013;70:1389-1395.

25. Mukherjee P, Chung SW, Berman JI, Hess CP, Henry RG. Diffusion tensor MR imaging and fiber tractography: technical considerations. AJNR Am J Neuroradiol 2008;29:843-852.

26. Zhang W, Li X, Zhang J, Luft A, Hanley DF, van Zijl P, et al. Landmark-referenced voxel-based analysis of diffusion tensor images of the brainstem white matter tracts: application in patients with middle cerebral artery stroke. Neuroimage 2009;44: 906-913.

27. Smith SM, Jenkinson M, Johansen-Berg $H$, Rueckert D, Nichols TE, Mackay CE, et al. Tract-based spatial statistics: voxe/wise analysis of multi-subject diffusion data. Neuroimage 2006; 31:1487-1505.

28. Zhang B, Xu Y, Zhu B, Kantarci K. The role of diffusion tensor imaging in detecting microstructural changes in prodromal Alzheimer's disease. CNS Neurosci Ther 2014;20:3-9.

29. Petersen RC. Mild cognitive impairment as a diagnostic entity. J Intern Med 2004;256:183-194.

30. Lee JH, Lee KU, Lee DY, Kim KW, Jhoo JH, Kim JH, et al. Development of the Korean version of the Consortium to Establish a Registry for Alzheimer's Disease Assessment Packet (CERAD-K): clinical and neuropsychological assessment batteries. J Gerontol B Psychol Sci Soc Sci 2002;57: P47-P53.

31. Morris JC. The Clinical Dementia Rating (CDR): current version and scoring rules. Neurology 1993;43:2412-2414.

32. Dale AM, Fischl B, Sereno Ml. Cortical surface-based analysis. 1. Segmentation and surface reconstruction. Neuroimage 1999;9:179-194.

33. Fischl B, van der Kouwe A, Destrieux C, Halgren E, Ségonne $\mathrm{F}$, Salat $\mathrm{DH}$, et al. Automatically parcellating the human cerebral cortex. Cereb Cortex 2004;14:11-22.

34. Fischl B, Salat DH, Busa E, Albert M, Dieterich M, Haselgrove
C, et al. Whole brain segmentation: automated labeling of neuroanatomical structures in the human brain. Neuron 2002;33:341-355.

35. Greve DN, Fischl B. Accurate and robust brain image alignment using boundary-based registration. Neuroimage 2009; 48:63-72.

36. Talairach J, Tournoux P. Co-planar stereotaxic atlas of the human brain : 3-dimensional proportional system: an approach to cerebral imaging. New York:Georg Thieme;1988. 122 p.

37. Behrens TE, Berg HJ, Jbabdi S, Rushworth MF, Woolrich MW. Probabilistic diffusion tractography with multiple fibre orientations: what can we gain? Neuroimage 2007;34:144-155.

38. Yendiki A, Panneck P, Srinivasan P, Stevens A, Zöllei L, Augustinack J, et al. Automated probabilistic reconstruction of white-matter pathways in health and disease using an atlas of the underlying anatomy. Front Neuroinform 2011,5:23.

39. Ezzati A, Katz MJ, Lipton ML, Zimmerman ME, Lipton RB. Hippocampal volume and cingulum bundle fractional anisotropy are independently associated with verbal memory in older adults. Brain Imaging Behav 2016;10:652-659.

40. Mori S, van Zijl PC. Fiber tracking: principles and strategies a technical review. NMR Biomed 2002;15:468-480.

41. Lee SH, Coutu JP, Wilkens P, Yendiki A, Rosas HD, Salat DH, et al. Tract-based analysis of white matter degeneration in Alzheimer's disease. Neuroscience 2015;301:79-89.

42. Brun A, Englund E. A white matter disorder in dementia of the Alzheimer type: a pathoanatomical study. Ann Neurol 1986; 19:253-262.

43. Kocahan S, Doğan Z. Mechanisms of Alzheimer's disease pathogenesis and prevention: the brain, neural pathology, $\mathrm{N}$-methyl-D-aspartate receptors, tau protein and other risk factors. Clin Psychopharmacol Neurosci 2017;15:1-8.

44. Mielke MM, Kozauer NA, Chan KC, George M, Toroney J, Zerrate $\mathrm{M}$, et al. Regionally-specific diffusion tensor imaging in mild cognitive impairment and Alzheimer's disease. Neuroimage 2009;46:47-55.

45. Wee CY, Yap PT, Zhang D, Denny K, Browndyke JN, Potter $\mathrm{GG}$, et al. Identification of $\mathrm{MCl}$ individuals using structural and functional connectivity networks. Neuroimage 2012;59: 2045-2056.

46. Kang DW, Lee CU, Lim HK. Role of sleep disturbance in the trajectory of Alzheimer's disease. Clin Psychopharmacol Neurosci 2017;15:89-99.

47. Seo EH, Lee DY, Lee JM, Park JS, Sohn BK, Lee DS, et al. Whole-brain functional networks in cognitively normal, mild cognitive impairment, and Alzheimer's disease. PLoS One 2013;8:e53922.

48. Petrella JR, Sheldon FC, Prince SE, Calhoun VD, Doraiswamy PM. Default mode network connectivity in stable vs progressive mild cognitive impairment. Neurology 2011;76:511517.

49. Binnewijzend MA, Schoonheim MM, Sanz-Arigita E, Wink AM, van der Flier WM, Tolboom N, et al. Resting-state fMRI 
changes in Alzheimer's disease and mild cognitive impairment. Neurobiol Aging 2012;33:2018-2028.

50. Ganguli M, Snitz BE, Saxton JA, Chang CC, Lee CW, Vander Bilt $\mathrm{J}$, et al. Outcomes of mild cognitive impairment by definition: a population study. Arch Neurol 2011;68:761-767.

51. Douet V, Chang L. Fornix as an imaging marker for episodic memory deficits in healthy aging and in various neurological disorders. Front Aging Neurosci 2015;6:343.

52. Kantarci K, Schwarz CG, Reid RI, Przybelski SA, Lesnick TG, Zuk SM, et al. White matter integrity determined with diffusion tensor imaging in older adults without dementia: influence of amyloid load and neurodegeneration. JAMA Neurol 2014;71:1547-1554. 